\title{
Ueber die Entwickelung der inneren und äusseren Genitalien beim menschlichen Weibe.
}

\author{
Von
}

Dr. W. Nagel, Berlin.

(Mit 6 Abbildnngen im Text.)

Es ist auffallend, wie wenig viele von den jüngeren Gynäkologen, welche sich berufen fühlen, durch eigene Untersuchungen zur Lösung anatomischer Fragen beizutragen, um die Arbeiten und Ansichten in den neueren anatomischen Fachschriften sich kümmern. Zum Zwecke der literarischen Verwerthung seiner Untersuchungen liest der junge Forscher vielfach nur die in der Zeitschrift für Geburtshülfe and Gynäkologie und im Archiv für Gynäkologie erschienenen Arbeiten und glaubt damit, wie es mir scheint, seinen Pflichten genügt zu haben; nicht einmal die von Hermann and Schwalbe so vortrefflich geleiteten Jahresberichte über die Fortschritte der Anatomie und Physiologie (Leipzig, Verlag von F. C. W. Vogel) werden zu Rathe gezogen. In Folge dessen findet man in vielen gynäkologisch-anatomischen Arbeiten der neueren Zeit immer noch alte Ansichten vertreten, die längst als unrichtig erkannt worden sind und Schlussfolgerungen angeführt, welchen die Grundlage durch moderne Untersuchungen entzogen worden ist. Trotzdem werden an diese veralteten und unrichtigen Ideen neue Schlüsse geknüpft: man tappt auf einem verlassenen, in falscher Richtung führenden Weg umher mit Hülfe von alten kaum leserlichen Wegweisern, und wird nicht gewahr, dass in einiger Entfernung ein gut gebahnter, wohlerhaltener Weg zum Ziele führt, der unablässlich von Fuhrwerken moderner Bauart befahren wird. 
Hat es schon gewisse Bedenken, wenn Anfänger ohne Leitung und ohne ständige kritische Beaufsichtigung von Seiten eines Fachmannes an die Lösung anatomischer Aufgaben herangehen, so wird die Sache doch recht bedenklich, wenn die Bearbeitung der gewonnenen Ergebnisse ohne Fühlung mit der anatomischen Fachliteratur geschieht. Man sehe sich nur in der anatomischen Jiteratur der letzten Jahre die fachmännische Kritik derartiger Arbeiten näher an und man wird His Recht geben müssen, wenn er sagt (Anatomie menschlicher Embryonen, Leipzig 1885, Einleitung), dass ein viel grösserer Nutzen für den Fortschritt der Wissenschaft entstehen würde, wenn derartige selbstständige Untersuchungen unterbleiben würden.

Dass im Beginne der neuen Aera der operativen Gynäkologie viele bis dahin völlig unbearbeitete Felder der Anatomie und Pathologie der weiblichen Genitalien mit Erfolg von Gynäkologen bearbeitet worden'sind, ist zur Genüge bekannt. Die Zeiten für derartige grundlegende Arbeiten sind jedoch jetzt vorüber.

Man hört ferner vielfach die Aeusserung, ,die bei Thieren gefundenen Thatsachen kann man nicht ohne weiteres auf Menschen überführen", und glaubt sich vielleicht dadurch der Mühe enthoben, die erwähnten Thatsachen überhaupt anzusehen.

Es mag ja richtig sein, dass auf gewissen Gebieten der $\mathrm{Pa}_{\mathrm{a}}$ thologie und der experimentellen Physiologie eine Ueberführung der gefundenen Thatsachen von Thieren auf den Menschen unstatthaft sei; was aber die Entwickelungsgeschichte betrifft, so ist dieser Satz ganz und gar ungültig. Die moderne Anatomie, vor Allem die Entwickelungsgeschichte, arbeitet unter dem Zeichen der vergleichenden Anatomie. Nur durch letztere Wissenschaft ist die Entwickelungsgeschichte überhaupt vorwärts gekommen; nur mit Hülfe der Biologie sind viele Probleme - ich erinnere z. B. an die Befruchtung - wenn auch nicht gelöst, so doch erfolgreich bearbeitet worden. Alle bisherigen Untersuchungen haben denn auch gezeigt, dass die Entwickelung des Menschen mit der der höheren Wirbelthiere in vielen Punkten übereinstimmt; ja, selbst mit niederen Thieren lassen sich noch auf jüngeren Entwickelungsstufen des Menschen unschwierig gewisse Parallele ziehen. Selbstverständlich kommen individuelle Abweichungen vor, im Allgemeinen muss aber dem Forscher Zweifel an die Richtigkeit seiner Beobachtungen aufkommen, wenn er sieht, dass diese mit den bei 
verwandten Thieren von zuverlässigen Beobachtern gefundenen Thatsachen in Widerspruch stehen.

Jedenfalls ist in entwickelungsgeschichtlichen Fragen eine eingehende Berücksichtigung der Fachliteratur für einen gedeihlichen Fortschritt dieser Wissenschaft unumgänglich nothwendig und um so mehr darf man sich wundern, dass dieses so wenig geschieht.

Hätte z. B. Popoff (Zur Morphologie und Histologie der Tuben und des Parovariums beim Menschen während des intraund extrauterinen Lebens bis zur Pubertät. Dieses Archiv Bd. 44, 2. Heft, 1893) in der neueren anatomischen Literatur sich umgesehen, so würde er seine literarische Angaben über die Entwickelung der inneren Geschlechtsorgane nicht mit Kölliker und Dohrn abgeschlossen haben and nicht hinzugefügt haben (1. c. S. 277): „die weiteren Untersuchungen über den Bau der Müller'schen Gänge im oberen Drittel derselben, d. h. in dem Abschnitte, welcher sich in die Fallopi'schen Tuben umwandelt, betreffen nur Beobachtungen aus den ersten Stunden oder ersten Tagen des intrauterinen Lebens. Diese Untersuchungen sind fast ausschliesslich an Thieren gemacht worden und lassen in Bezug auf ihre weitere Ausarbeitung noch vieles zu wünschen übrig" und (l. c. S. 297): ,die Anatomie des Parovariums ist bis auf den heutigen Tag noch nicht genügend aufgeklärt; alle diesbezüglichen Arbeiten gehören meistens einer längst vergangenen Zeit an, wo die Kenntniss von den Geweben und ihrer technischen Bearbeitung noch nicht auf der Stufe stand, welche wir jetzt erreicht haben."

Popoff würde im Gegentheil viele und ausgezeichnete Arbeiten über die Entwickelung der Genitalien nicht allein bei Thieren, sondern auch beim Menschen gefunden haben, so z. B.: yon Mihalkovicz, Untersuchungen über die Entwickelung des Harnund Geschlechtsapparates der Amnioten. Internationale Monatsschrift für Anatomie und Histologie Bd. II, Paris, Leipzig, London 1885. van Ackeren, Beiträge zur Entwickelungsgeschichte der weiblichen Sexualorgane des Menschen. Inaugural-Dissertation. Zeitschrift für wissenschaftliche Zoologie Bd. 48. J. Janosik, Histologisch-embryologische Untersuchungen über das Urogenitalsystem. XCI. Band der Sitzungsberichte der kaiserlichen Akademie der Wissenschaften. Wien 1887. Tourneux et Legay, Memoire sur le developpement de l' Uterus et du Vagin. Journal de l'anatomie et de la Physiologí 1884. Tourneux, L'organ de Rosenmüller (Epoophore) et le Parovarium (Paroophore) chez les 
mammifères. Journal de l'anatomie et de Physiologie. 1884. C. K. Hoffmann, Zur Entwickelungsgeschichte der Urogenitalorgane bei den Reptilien. Zeitschrift für wissenschaftliche Zoologie Bd. 48, 1889. Wiedersheim, Ueber die Entwickelung des Urogenitalapparates bei Krokodilen und Schildkröten. A. f. mikroskop. Anatomie Bd. 36, 1890. Ballantyne and Williams, The Structures in the Mesosalpinx. Edinburgh 1893 (als „Histology and Pathology of the Fallopian Tube" erschienen im British Medical-Journal 1891, No. 1568 und No. 1569), und es würde ihm wohl nicht entgangen sein, dass auch ich seit mehreren Jahren (im Laboratorium des I. anatomischen Instituts zu Berlin) mit der Entwickelung des weiblichen Genitalapparates beim Menschen mich eingehend beschäftige.

Eine kürzere Mittheilung dieser meiner Untersuchungen ist jedesmal von Waldeyer in der Königl. Preuss. Akademie der Wissenschaften niedergelegt worden (siehe die Sitzungsberichte der Akademie von 1887, 1888, 1889, 1890, 1891, 1892).

Die ausführliche Darlegung meiner Ansichten habe ich abgesehen von den Veröffentlichungen in diesem Archiv - in folgenden Aufsätzen niedergelegt: „Das menschliche Ei,“ Arch. für mikroskop. Anatomie Bd. 31, 1888. „Ueber die Entwickelung des Urogenitalsystems des Menschen", ibidem Bd. 34, 1889. "Ueber die Entwickelung des Uterus und der Vagina beim Menschen," ibidem Bd. 37, 1891. "Ueber die Entwickelung der Urethra und des Dammes beim Menschen," ibidem Bd. 40, 1892.

Es liegt mir fern, den Werth der Untersuchungen Popoff's oder anderer herabzusetzen; ich muss aber bemerken, dass ich meine Untersuchungen an einer ganz anderen vollständigen und vollzähligen Reihe mehrerer Hundert menschlicher Embryonen, welche ich zum allergrössten Theil meinem hochverehrten Chef, Herrn Professor Gusserow, verdanke, angestellt habe, als z. B. Popoff; auch die Entwickelung des Parovariums und der Tube. ist von mir in Wort und Bild dargestellt worden. Da meine Arbeiten eingehende Beachtung ron Seiten anatomischer Fachgenossen im In- und Auslande erfahren haben, da sie ferner in den betreffenden anatomischen Fachjournalen stets ausführlich referirt werden, so darf ich wohl erwarten, dass sie auch von denjenigen gynäkologischen Forschern berücksichtigt werden, welche sich mit diesem Gegenstande beschäftigen werden. Um dieses zu erleich- 
tern, gestatte ich mir an diesem Orte eine Uebersicht meiner Beolachtungen zu geben.

\section{Die Geschlechtsdrüsen.}

Bei menschlichen Embryonen von 11-13 mm Länge ist die Keimdrüse eben angelegt und zeichnet sich als ein $0,5 \mathrm{~mm}$ langer und $0,3 \mathrm{~mm}$ breiter Wulst an der Innenseite des Wolff'schen Körpers an derselben Stelle, wo auch bei den meisten übrigen Wirbelthieren die erste Anlage der Sexualdrüsen zu sehen ist (Waldeyer).

Schon auf dieser Entwickelungsstufe vermag man (an den Keimdrüsen) das männliche von dem weiblichen Geschlecht zu trennen.

Die eben angelegte Sexualdrüse besteht der Hauptsache nach aus epithelialen Elementen, welche gegen das Stromagewebe des Wolff' schen Körpers deutlich abzugrenzen sind. In diesem Keimepithelwulst bemerkt man deutlich bei einigen Embryonen stellenweise eine gewisse regelmässige Anordnung der Zellen; sie bilden geschlängelte Stränge, welche theils unter sich, theils mit der Oberfläche in Verbindüng stehen. Zwischen den genannten Strängen erkennt man, aber nur an der Basis des Organs, spärliohe Züge von zartem, embryonalem Bindegewebe mit spindelförmigen Zellen. Ferner sieht man über das ganze Organ vertheilt, einzelne grosse Zellen, die sofort durch ihren blassen $8 \mu$ grossen Kern in die Augen fallen. - Das ist die erste Anlage des Hodens; die grossen Zellen sind die Ursamenzellen.

Bei anderen menschlichen Embryonen derselben Grösse bietet der Keimepithelwulst ein ganz anderes Aussehen: hier ist von einer Anordnung der Zellen zu Strängen nicht die Rede; es lässt sich überhaupt keine bestimmte Anordnung der Zellen erkennen und die zarten Züge embryonalen Bindegewebes, welche bei den ersterwähnten deutlich an der Basis des Organs zu erkennen waren, sind hier wohl an einzelnen Stellen vorhanden, aber mehr zurücktretend. Ganz auffallend ist aber, dass die grossen Zellen bei den hier in Rede stehenden Embryonen viel zahlreicher auftreten, als bei den ersterwähnten. Besonders ist dieses auffallend bei einer Durchmusterung ganzer Schnittserien. Ausserdem sind die Zellen grösser und lassen meist ein deutliches Protoplasma erkennen. Jede dieser Zellen misst bis zu $16 \mu$; auch der Kern ist etwas 
grösser als der der Ursamenzellen $(9 \mu)$ und lässt meistens ein deutliches Kerngerüst erkennen. Das ist die erste Anlage des Eierstockes; die grossen Zellen sind die Ureier.

Die weitere Entwickelung der Sexualdrüsen ist ferner bei beiden Geschlechtern von Anfang an eine so verschiedene, dass es berechtigt erscheint anzunehmen, dass eine Trennung des Geschlechts schon in sehr frühen Stadien, vielleicht von Anfang an stattfindet; sobald die Keimdrüse als solche angelegt ist, kann man auch an dieser unterscheiden, ob der Embryo männlich oder weiblich ist.

Betrachten wir zunächst den Hoden.

Die vorhin erwähnten Zellstränge wachsen theils durch Vermehrung ihrer eigenen zelligen Elemente, theils dadurch, dass die Keimepithelzellen (mit den aus ihnen herrorgegangenen Ursamenzellen), welche, wie gesagt, den Hauptbestandtheil des Geschlechtswulstes bilden, sich immer mehr und mehr zu Strängen ordnen. Der letzte Vorgang ist die Hauptquelle für die Vermehrung der Zellstränge. Irgend eine Betheiligung von Seiten der Wolff'schen Canäle (Malpighi'schen Körperchen), eine Einwanderung von Zellbalken (Segmentalstränge) im Sinne von Balfour and Braun findet hierbei nicht statt. Schritt haltend mit der Wucherung der zelligen Elemente des Keimepithelwulstes wachsen vom Gewebe des Wolff'schen Körpers junge Bindegewebszellen in den Keimepithelwulst hinein und führen alsbald zur Bildung embryonalen Bindegewebes. An zwei Stellen tritt besonders früh die Bindegewebsbildung auf, nämlich dicht unterhalb der Oberfläche des Wulstes und zwischen den Zellsträngen: hierdurch erhält der Hoden ziemlich früh den für ihn eigenthümlichen Bau. An Embryonen von $18-22 \mathrm{~mm}$ Länge erkennt man eine deutliche, aus embryonalem Bindegewebe bestehende Albuginea, durch welche die Zellstränge, die späteren Samencanälchen, vollständig von dem Oberflächenepithel, welches jetzt aus regelmässigen cubischen Zellen besteht, getrennt werden. Auf dieser Entwickelungsstufe bestehen die Zellstränge wesentlich aus regelmässig angeordneten langcylindrischen, nach der Mitte des Stranges, dem späteren Lumen, spitz zulaufenden Zellen mit einem dentlichen länglichen Kern. In den Zellsträngen zerstreut sieht man die vorhin erwähnten grossen Zellen, die Ursamenzellen.

Sobald die Zellstränge durch das embryonale Bindegewebe von dem Oberflächenepithel getrennt sind, wachsen sie nur durch Ver- 
mehrung ihrer eigenen zelligen Elemente und das Oberflächenepithel verhält sich, als einfaches Deckepithel, vollkommen passiv der weiteren Entwickelung des Hodens gegenüber. Das Oberflächenepithel des fötalen Hodens (das Hodenepithel) ist also nur als ein Rest des Keimepithels, als die äusserste Schicht des Keimepithelwulstes zu betrachten.

Die sogenannten $\mathrm{Z}$ wischensubstanzzellen, die sich durch ihren Protoplasmareichthum auszeichnen, habe ich zuerst bei einem Embryo von $31 / 2 \mathrm{~cm}$ Länge gesehen. Im Laufe der weiteren Entwickelung, wenigstens noch bei Embryonen von $12 \mathrm{~cm}$ Kopf-Steisslänge, nimmt die Zwischensubstanz an Mächtigkeit zu, wodurch die noch immer soliden Samencanälchen weiter auseinandergedrängt werden. Die spätere Entwickelung des Hodens ist mit der Frage der Spermatogenese eng verknüpft und ist von anderen Forschern (Benda, Klaatsch, His, van Beneden u. A.) beschrieben worden.

Aehnlich wie der Hoden kennzeichnet sich der Eierstock in seiner ersten Anlage auch als ein Keimepithelwulst, welcher sich aber dadurch von dem männlichen Epithelwulst unterscheidet, dass eine weit grössere Menge Keimepithelzellen sich in grosse Geschlechtszellen (Ureier) umwandelt, und dass die Zellen niemals, auf keiner Stufe der Entwickelung, eine derartige schlauchähnliche Anordnung annehmen wie in der Anlage des Hodens. Viel später als beim Hoden und viel langsamer wuchern die Bindegewebszellen von dem Stroma des Wolff'schen Körpers (ohne Betheiligung der Wolff'schen Canäle) herkommend, in den Keimepithelwulst hinein und zerlegen durch Bildung von Bindegewebe diesen, und zwar die tieferen Schichten desselben, in die Eifächer (Eiballen Waldeyer, Keimfächer Pflüger). Zur Bildung einer Albuginea kommt es nicht: die oberste Schicht des Keimepithelwulstes bleibt bis zur beendeten Bildung des Primärfollikel in ständiger Verbindung mit den tieferen Zellschichten.

Das Wachsthum der zelligen Elemente geschieht hauptsächlich an der Oberfläche der Eierstocksanlage. "Durch starke Vermehrung der Keimepithelzellen in den oberen Schichten und Umwandelung eines grossen Theiles dieser in Ureier baut sich der Eierstock auf; Schritt haltend hiermit wächst das junge Bindegewebe aus der Tiefe empor und zerlegt die neugebildeten Epithelmassen nach und nach in Eiballen. Die jüngsten Stufen der Entwickelung findet man also stets an der Oberfläche der Eierstocksanlage. 
Die Bildung von Primärfollikeln (Urei umgeben mit einem einschichtigen Follikelepithel) tritt zuerst in den tiefer liegenden Schichten auf, indem die Eifächer durch hereinwachsendes Bindegewebe noch weiter zerlegt werden. Schritthaltend mit dem Wachsen des Ovariums wird die Follikelbildung immer mehr nach der Peripherie verlegt, so dass die der Oberfläche des Ovariums zunächst liegenden Eiballen zuletzt in Primärfollikel umgebildet werden. In dem fertig gebildeten Ovarium - am Ende des intrauterinen Lebens - sieht man denn häufig eine Verbindung der jüngsten Follikel mit dem Keimepithel, wie die Eizellen geradezu in Einstülpungen (Valentin-Pflüger'sche Schläuche) von dem Keimepithel ausgehehend, liegen. Es sind diese Einstülpungen Ueberbleibsel der Eifächer und ein schlagender Beweis dafür, dass das Follikelepithel wirklich aus den Zellen des Keimepithels hervorgeht (Waldeyer).

\section{Die Wolff'schen Körper.}

In dem Wolff'schen Körper (Urniere) unterscheidet man die Glomeruli (Malpighi'sche Körperchen) die Urnierencanälchen und den Wolff'schen Gang, welcher das Sammelrohr der Canälchen und das Abflussrohr der Urniere darstellt.

Bei jüngeren menschlichen Embryonen ist das Epithel ein gleichartiges im ganzen Bereiche eines jeden Urnierencanälchens. Bei Embryonen von 18-23 mm tritt hierin eine sehr wesentliche Aenderung ein, indem das Epithel in dem weiten, in die $\mathrm{Bow}-$ man'schen Kapseln unmittelbar übergehenden Anfangsstücke eines jeden Canälehens jetzt aus grossen fast cubischen Zellen mit hellem Protoplasma und blassem Kern besteht. Bei einem Embryo von $18 \mathrm{~mm}$ fand ich, dass die freie Fläche dieser Zellen - nach der Lichtung des Canälchens zu - kurze Fortsätze trug. Wahrscheinlich sind diese Fortsätze als Gilien aufzufassen, um so mehr, als Janosik, Nicolas, v. Mihalkovicz und Wiedersheim bei Säugethieren und Reptilien Flimmerhaare an den erwähnten Zellen gesehen haben. Die grossen Zellen treten zu einer Zeit auf, wo die von dem embryonalen Stoffwechsel an den Secretionsapparat der Urniere gestellten Ansprüche in Steigerung begriffen sind und verschwinden vollkommen, sobald die Urnieren durch die heranwachsenden Nieren entlastet worden sind; sie bilden eine Eigenthümlichkeit des Wolff'schen Körpers zur Zeit seiner höchsten 
Entwickelung und Thätigkeit. Wahrscheinlich ist die von von Mihalkovicz und Nicolas für die Säugethiere gegebene Erklärung, dass nämlich die mit den erwähnten grossen Zellen bekleideten Abschnitte zu dem secretorischen Apparate der Urniere gehören, auch für den Menschen zutreffend.

Mit Waldeyer trennt man den Wolff'schen Körper in einen „Sexual“- und einen „Urnierentheil"; es ist auch für den Menschen erwiesen (Kobelt, Waldeyer, Kölliker, Gasser) und auch aus meinen Untersuchungen geht es hervor, dass der Nebenhoden aus dem proximalen Abschnitte (Sexualtheil) der Urniere hervorgeht. Die besondere Lage der Glomeruli, nämlich in dem medialen Theile der Urniere, trägt bei weiblichen Embryonen zu einer noch deutlicheren Trennung der Urniere in zwei Abschnitten bei, wie meine abgebildeten Präparate zeigen (a. a. 0.). Bei älteren Embryonen wird diese Trennung der Urniere in zwei Abschnitte noch mehr auffällig, indem die Glomeruli, sowohl bei männlichen als bei-weiblichen Individuen in dem proximalen Theile zuerst schwinden, während sie in dem mittleren und distalen Abschnitte viel länger erhalten bleiben.

Gasser hat z. B. noch bei männlichen Embryonen aus dem VI. Monate, Kölliker bei solchen aus dem IV. Monate deutliche Glomeruli im unteren Theile der Urniere gefunden. Auch ich habe bei allen meinen Embryonen, ohne Rücksicht auf das Geschlecht, deutliche Glomeruli im distalen Ende gesehen bis zu Ende des IV. Monats und Spuren derselben lassen sich noch, wenigstens bei den weiblichen Individuen, in den späteren Monaten der Schwangerschaft nachweisen, was auch Waldeyer und Tourneux hervorheben. Diesen beiden Forschern zufolge findet man bei weiblichen Individuen nur ausnahmsweise in dem extrauterinen Leben Ueberreste dieses „Urnierentheils" des Wolff'schen Körpers (Paroophoron (Waldeyer), gleichwerthig mit Paradidymis oder corps innominé Giraldés beim Manne), während bekanntlich der „Sexualtheil“ (Epoophoron (Waldeyer), Rosenmüller's Organ, Parovarium im alten Sinne des Wortes, gleichwerthig mit Epididymis beim Manne) ein beständiges Anhängsel des Eierstockes bleibt. Nach den neuesten Untersuchungen von Tourneux wächst das Epoophoron mit dem Alter des Individuums; erst nach der Menopause beginnt es zu atrophiren. Die Ueberreste des Urnierentheils (Paroophoron) sind wahrscheinlich die Bildungsstätten der sogenannten Cysten des breiten Mutterbandes (Waldeyer). 


\section{Die Müller'schen Gänge.}

Aus meinen Untersuchungen geht hervor, dass der Müller'sche Gang bei ganz jungen menschlichen Embryonen (von 8 bis $13 \mathrm{~mm}$ Länge) beiderlei Geschlechts als eine trichterförmige (richtiger ,dütenförmige") Einstülpung des verdickten Coelomepithels am proximalen Theil der Urniere angelegt wird, welche mit ihrem soliden, spitz zulaufenden Ende dem Wolff' schen Gange dicht anliegt, wobei eine innige Verbindung des Epithels der beiden Gebilde eintritt, jedoch ohne dass es, wie es scheint, zu einer Verschmelzung kommt. Die Anlegung des Müller'schen Ganges geschieht also beim Menschen in derselben Weise, wie bei Säugethieren, Vögeln und Reptilien (Bornhaupt, Waldeyer, Egli, Gasser, Balfour, Sedgwick, Janosik, von Mihalkovicz, Wiedersheim). Während der weiteren Entwickelung wächst der Müller'sche Gang (beim Menschen) der ventralen Wand des Wolff'schen Ganges entlang abwärts (er benutzt, so zu sagen, den Wolff'schen Gang als Leiter) bis er den Sinus urogenitalis erreicht. Dadurch, dass die ventrale Kante der Urniere durch eine spiralige Drehung des ganzen Organs nach innen, das heisst nach der Längenachse des embryonalen Körpers zu, allmälig umbiegt, wird es erklärlich, weshalb der Müller'sche Gang, der in dem proximalen Theile der Urniere nach aussen von dem Wolff'schen liegt, in dem distalen Theile des Organs, in der Plica urogenitalis (Waldeyer) und in dem Genitalstrange (Thiersch) nach innen von dem genannten Gange belegen ist. Auf der letzgenannten Strecke liegen die beiden Müller'schen Gänge dicht aneinander; ihre nach innen liegenden Wandungen berühren sich und werden gleichzeitig an mehreren Stellen durchbrochen; durch diesen Vorgang entsteht schliesslich aus den ursprünglich getrennten Gängen ein einziger Hohlraum (Dohrn). Ferner habe ich nachgewiesen, dass der solide Endzapfen des Müller'schen Ganges aus eigenartigen cubischen protoplasmareichen Zellen besteht, ganz gleich, ob es sich um weibliche oder männliche Individuen handelt. Während des Abwärtswachsens des Müller'schen Ganges ändern die genannten Zellen ihr Aussehen nicht; in dieser ganzen $\mathrm{Pe-}$ riode, von der ersten Anlage des Ganges als röhrenförmiges Gebilde bis zur Erreichung des Sinus urogenitalis, kann man vielmehr zwei Abtheilungen an demselben deutlich von einander unterscheiden: nämlich einen proximalen (grösseren) 
Abschnitt, welcher ein deutliches Lumen besitzt und dessen Wände mit hohen und schmalen Cylinderzellen ausgekleidet sind, und einen distalen (kleineren), welcher keine Höhlung besitzt und aus den oben gedachten grossen Zellen von vorwiegend cubischer Gestalt gebildet wird.

Dieser Unterschied in der epithelialen Auskleidung besteht auch noch fort, nachdem die Müller'schen Gänge den Sinus urogenitalis erreicht haben. Zahlreiche Schnittreihen in den oben erwähnten Körperrichtungen durch das Becken von menschlichen Embryonen, weiblichen Geschlechts, mit einer Rumpflänge von $3-4 \frac{1}{2} \mathrm{~cm}$ haben übereinstimmend folgenden Befund gegeben (siehe meine a. a. 0. abgebildeten Präparate): Der Geschlechtsstrang und die angrenzenden Theile der Plicae urogenitales (der späteren uterinen Abschnitte der Tuben mit angrenzendem Stück der Ligamenta lata) zeigen eine seichte dorso-ventrale Krümmung mit vorderer Concavität. An seinem proximalen Ende ist der Geschlechtsstrang gabelförmig getheilt, während sein distales Ende etwas spitz zulaufend die hintere Wand des Sinus urogenitalis in schräger Richtung durchbohrt und in diesen hügelartig hineinragt; die hierdurch entstehende Erhabenheit hat v. Mihalkovicz mit dem Namen "Müller'scher Hügel" belegt. In seinem mittleren Theile zeigt der Geschlechtsstrang eine gleichmässige spindelförmige Verdickung und umschliesst folgende epitheliale Gebilde: 1. Die Müller'schen Gänge. Das proximale (längere) Stück ist mit hohem schmalen Cylinderepithel ausgekleidet und besitzt ein deutliches Lumen, das distale (kürzere) Stück ist ausgefüllt mit den oben beschriebenen grossen protoplasmareichen Zellen, welche rundliche Kerne tragen und im Uebrigen dasselbe Verhalten zeigen wie auf früheren Entwicklungsstufen. Dieses distale Stück hat keine Höhle und vermittelt also die Verbindung des Geschlechtsganges mit dem Sinus urogenitalis. 2. Die Wolff'schen Gänge. Dieselben sind bis zu ihrer Einmündung in den Sinus urogenitalis deutlich von den Müller'schen Gängen abzugrenzen, obwohl die Gänge distalwärts einander dicht anliegen. Um diese Zeit findet sich die Mündung der Wolff'schen Gänge in den Sinus urogenitalis dicht neben der Einmündung der Müller'schen Gänge und in gleicher Höhe.

Die eben besprochene Krümmung des Geschlechtsstranges (deren Entstehung ich anderweit - dieses Archiv, Bd. 41 [Festschrift zu Ehren Gusserow's], „Ueber die Lage des Uterus im 
menschlichen Embryo" - geschildert habe) gestaltet sich zuerst als ein naheza gleichmässig verlanfender Abschnitt eines Kreises; dessen Centrum man sich ausserhalb des Körpers, etwas oberhalb der Symphysis pubis zu denken hat und an welchem man etwa in der Mitte eine flaçhwinkelige Knickungsstelle bemerkt. Spätere Entwicklungsstufen zeigen, dass dieser Knickungswinkel im Lumen des Geschlechtsstranges derjenigen Stelle entspricht, wo der äussere Muttermund sich bildet. Bei grösseren Embryonen (mit einer. Kopf-Steisslänge von 6 bis $10 \mathrm{~cm}$ und darüber) gesellt sich noch eine besondere Neigung des oberen Abschnittes des Geschlechtsstranges nach vorn hinzu, welche mitunter so stark wird, dass das (spätere) Corpus uteri horizontal liegt; der hierdurch entstandene, von dem vorher genannten wohl za unterscheidende neue Knickungswinkel liegt in der Gegend des (späteren) inneren Muttermundes.

Es besteht also nach meinen Beobachtungen von vornherein ein Unterschied in der Epithelbekleidung des proximalen und distalen Abschnittes des Geschlechtsstranges: in dem erstgenannten (der spätere Uterus) wird das Epithel aus hohen und schmalen Cylinderzellen mit länglichen Kernen gebildet, in dem letztgenannten (die spätere Vagina) sind dio Epithelzellen kürzer, breiter und haben rundliche Kerne. Dieser Unterschied der Epithelbekleidung ist besonders in die Augen fallend, wenn man die beiden oben erwähnten Abschnitte des Geschlechtsstranges mit einander vergleicht; jedoch ist der Uebergang zwischen den beiden. Epithelarten kein scharfer, plötzlicher; derselbe vollzieht sich vielmehr allmälig innerhalb einer Strecke von einigen Mikromillimetern; je älter der Embryo, um so schärfer tritt jedoch der Epithelunterschied hervor. Aus den späteren Entwicklungsstufen (bei Embryonen mit einer Kopf-Steisslänge von $17 \mathrm{~cm}$ und darüber) ist zu entnehmen, dass die Uebergangsstelle gleichwerthig ist mit derjenigen Stelle etwas oberhalb des äusseren Muttermundes, wo unter normalen Verhältnissen im späteren Cervicalkanale die Grenze zwischen dem Cylinderepithel des Uterus und dem Plattenepithel der Vagina zu sehen ist. Es findet also an keiner Stelle des Geschlechtsstranges und auf keiner Entwicklungsstufe eine Umwandlung von Cylinderepithel in Plattenepithel statt, wie man anzunehmen scheint (Tourneux). Es besteht vielmehr nach meinen Untersuchungen von vornherein und während der ganzen Entwicklung eine Trennung der beiden Abschnitte des 
Geschlechtsstranges nicht allein durch den erwähnten Epithelunterschied, sondern auch durch den mehr gestreckten Verlauf (nach vorn unten) des distalen Theiles und durch die Beschaffenheit der umliegenden mesodermalen Bildungszellen. Aus diesem distalen Theile wird also - durch einfaches Längenwachsthum die Vagina gebildet.

Auch zwischen dem Bereich des späteren Corpus und dem Bereich der Cervix uteri treten schon sehr früh deutliche Epithelunterschiede auf, im Corpusbereich ist das cylindrische Epithel niedriger als im Bereich des späteren Cervicalkanals, wo es mehrschichtig wird und auf Längsschnitten ein wellenförmiges, später sägeförmiges (Anlage der Cervicaldrüsen) Aussehen zeigt.

Die endgültige Trennung zwischen Uterus und Vagina beginnt durch die Bildung der Portio vaginalis bei Embryonen mit einer Kopfsteisslänge von $10-14 \mathrm{~cm}$. Zuerst geschieht die Anlage und Abgrenzung der hinteren Muttermundslippe dadurch, dass etwas unterhalb der oben erwähnten Epithelgrenze das Epithel in Gestalt eines Zapfens in die hintere Wand des Geschlechtsstranges einwuchert und zwar etwas schräg nach oben. Um diese Zieit (zuweilen auch früher) deutet eine buckelige Hervorragung der vorderen Wand des Geschlechtsstranges nach dem Lumen zu diejenige Stelle an, wo sich später die vordere Muttermundslippe bildet, und zwar durch einen ähnlichen Vorgang, wie oben beschrieben (vergl. die Arbeiten von Dohrn, v. Baer, v. Kölliker, van Ackeren, v. Mihalkoricz, Tourneux et Legay). Den Bildungsvorgang der Portio vaginalis kann man folgendermaassen zusammenfassen: die vordere Muttermundslippe wird zuweilen zuerst angelegt, jedoch entwickelt sich in der Regel das hintere Scheidengewölbe früher als das vordere, und die hintere Muttermundslippe erlangt dadurch früher als die vordere eine vollkommene Ausbildung.

Während dieser Vorgänge sinken die Eierstöcke und die Tuben in das kleine Becken herab und der Wolff'sche Körper bildet sich zurück (siehe S. 461). Die Tuba zeigt ungefähr in ihrer Mitte eine stumpfwinklige Knickung und ausserdem mehrere seichte Faltungen, welche dadurch entstehen, dass die Tuben durch das Herabsinken der mit ihnen eng verbundenen Eierstöcke verhindert werden, der Länge nach sich auszudehnen. Auf dem Querschnitt zeigen die Tuben ein ähnliches Verhalten wie andere Körperkanäle des Embryos auf dieser Entwicklungsstufe: um die mit einschichtigem Cylinderepithel ausgekleideteHöhlung zeigen die Bil- 
dungszellen eine circuläre Anordnung und stehen hier dichter als in der äusseren Schicht der Wand. Um diese Zeit zeigt ferner die fötale Tube eine halbe spiralige Drehung um die Längsaxe. Die Entstehung dieser Drehung lässt sich auf eine sehr frühe Entwicklungsstufe zurückführen und wird durch den eigenthümlichen Verlauf der Wolff'schen Gänge bedingt. Die ventralen Kanten der Urniere, in welcher der Wolff'sche Gang verläuft, biegen nämlich fusswärts allmälig nach innen, d. h. nach der Längenaxe des embryonalen Körpers, um und vereinigen sich schliesslich zur Bildung des Geschlechtsstranges (im Sinne von Thiersch). Da nun der Müller'sche Gang der ventralen Wand des Wolff'schen Ganges entiang abwärts wächst (siehe S. 462), so muss er dieselbe spiralige Drehung machen wie dieser und liegt infolge dessen, wie bekannt, in dem proximalen Theile der Urniere nach aussen, in der Plica urogenitalis und in dem Geschlechtsstrange nach innen von dem Wolff'schen Gange. Diese halbspiralige Drehnng besteht also lange, ehe die Eierstöcke sich in das Becken herabzusenken beginnen und ich vermag Freund (Ueber die Indikationen zur operativen Behandlung der erkrankten Tuben. Volkmann's Sammlung klin. Vorträge, No. 323, 1888) nicht beizupflichten, wenn er sagt, dass spiralige Drehungen (sechs und eine halbe, selten sieben und eine halbe) während der Herabwanderung der Tuben und der Eierstöcke in das kleine Becken entstehen. Tube und Eierstock sind um diese Zeit, während der sogenannten Herabwanderung, mittelst der Wolff'schen Körper und der Plicae urogenitales (aus deren bindegewebigen Elementen bekanntlich die Ligamenta lata, Ligamenta ovarii u. s. w. entstehen) an die hintere seitliche Wand der Bauchhöhle festgeheftet, so dass anch theoretisch die Entstehung von spiraligen Drehungen unmöglich ist.

Das Herabtreten der Eierstöcke und der Tuben, worunter man sich keine aktive Wanderung, sondern ein ungleichmässiges Wachsthum vorzustellen hat, ist bei Embryonen von 4-6-8 cm Rumpflänge vollzogen (also im 3. Monat), denn um diese Zeit berührt die untere Spitze der Eierstöcke die hintere Wand des Fundus uteri. Von jetzt an verschwindet also das Moment, welches nach Freund die spiraligen Drehungen herrorruft und auch jetzt noch lässt sich nur eine Halbspirale nachweisen, dagegen zeigt die Tube mehr oder weniger ausgesprochene einfache Faltungen, deren Entstehung wohl auf dem oben angedeuteten ungleichmässigen Wachsthum beruht, welches auch auf das Verhältniss ron Eierstock zu 
Tube sich bezieht. Hervorheben möchte ich noch, dass man an den Tuben (ebenso wie an den Eierstöcken) aus dieser Entwickelungsperiode Eindrücke von den umliegenden Organen bemerkt, wodurch ihre Oberfläche ein ungleichmässiges Aussehen erhält. Diese Eindrücke können unter Umständen Windungen vortäuschen.

Bei Föten aus den letzten 2 Schwangerschaftsmonaten und bei Neugeborenen finde ich dagegen, dass die Faltungen bei weitem am zahlreichsten und am deutlichsten sind. Es kommen um diese Zeit an den Tuben alle die Formen von Falten und Knickungen vor, welche überall entstehen, wenn ein elastisches Rohr zusammengelegt wird, um in einem verhältnissmässig zu kleinen Behälter Platz zu finden, zuweilen auch - begünstigt durch die aus der ersten Entwickelungsperiode stammende Halbspirale wirkliche korkzieherartige Windungen, obwohl nicht in der Regelmässigkeit wie Freund angiebt. $\mathrm{Da}$, wie gesagt, die Knickungen und Windungen erst lange nachdem die Eierstöcke in das kleine Becken herabgetreten sind, sich ausbilden, so ist ihre Entstehung nur auf ein isolirtes stärkeres Wachsthum der Tube zurückzuführen.

Die Mündungsstelle der Müller'schen Gänge in den Sinus urogenitalis (Canalis urogenitalis nach Rathke) jüngerer Embryonen ist gleichwerthig mit dem Introitus vaginae älterer Embryonen und Erwachsener. Sobald also die Müller'schen Gänge in der oben beschriebenen Weise den Canalis urogenitalis erreicht haben (bei Embryonen von $3 \mathrm{~cm}$ Rumpflänge), ist das Orificium vaginae vestibulare gegeben. Mit Kölliker und v. Mihalkovicz kann man von diesem Augenblicke an von einer Anlage des Hymen sprechen. Das Orificium ist auf allen Entwicklungsstufen, wenigstens bis zu einer Grösse des Embryo von $20-22 \mathrm{~cm}$ Kopfsteișslänge mit Epithelien ausgefüllt; von einem Durchbruch einer abschliessenden bindegewebigen Membran ist aber nicht die Rede.

Die anfänglich sehr kurze Scheide wächst später bedeutend in die Länge. Es findet nun in der Vagina eine eigenthümliche Anhäufung von Epithel statt und zwar zunächst dicht oberhalb der Mündung in den Sinus urogenitalis, wodurch die Vagina an dieser Stelle (bei Embryonen von 10-12 cm Kopfsteisslänge und darüber) eine bauchige oder blasige Erweiterung erfährt:' Durch diese Erweiterung ist die erste Anlage des Hymen gegeben. Da nämlich die Ränder der ursprünglichen Mündung von der Erweiterung 
nicht betroffen werden, da die Mündung vielmehr ihre anfängliche Enge behält und die Erweiterung oberhalb derselben liegt, so muss sich in dieser Weise ein Ring bilden, durch welchen die Vagina von dem Sinus urogenitalis abgeschlossen wird. In gewissen Fällen mag auf einer späteren Entwicklungsstufe noch ein aktives Wachsthum, besonders der hinteren Scheidenklappe stattfinden, wodurch die Hymenalöffnung theils enger, theils mehr nach vorn verlegt wird.

Bei Embryonen von $21 \mathrm{~cm}$ Rumpflänge ist die Bildung der Portio vaginalis uteri beendet and der Canalis urogenitalis za Vestibulum vaginae geworden.

Schaeffer (Bildungsanomalien weiblicher Geschlechtsorgane aus dem fötalen Lebensalter mit besonderer Berücksichtigung der Entwickiung des Hymens. Dieses Archiv, Bd. 37, S. 199) hat die Entwicklung des Hymen an Föten aus der letzten Hälfte der Schwangerschaft untersucht. $\mathrm{Da}$ indessen die oben geschilderten Vorgänge bei der Anlage des Hymen bei Embryonen von 3 bis $14 \mathrm{~cm}$ Körperlänge sich abspielen, so ist es klar, dass Embryonen. aus der letzten Hälfte keinen Aufschluss über diese Frage zu geben vermögen, weil das Hymen um diese Zeit längst gebildet ist.

Die Verödung des distalen Endes der Wolff'schen Gänge fängt bei weiblichen Embryonen zur selben Zeit an, wenn das distale Ende der vereinigten Müller'schen Gänge den Canalis urogenitalis erreicht hat und nun in die Länge zu wachsen beginnt. Die Function der Wolff'schen Gänge ist um diese Zeit (bei Embryonen von 4-5 cm Rumpflänge) beendigt; sie betheiligen sich nicht mehr an der weiteren Entwicklung des Geschlechtsstranges und somit ist es erklärlich, dass man in der Regel unterhalb des vorhin beschriebenen Epithelüberganges in dem Geschlechtsgange (also im Bereiche des späteren Collum uteri) keine Spur der Wolff'schen Gänge mehr findet. - Mit Dohrn (Die Gartner'schen Kanäle beim Weibe. Dieses Archiv, Bd. 21, 1883), bei dem man einen historischen Ueberblick dieser Frage findet, mit Rieder, Kölliker, Waldeyer u. A. bin ich darin einverstanden, dass man die Reste der Wolff'schen Kanäle (Gartner'sche Gänge) fusswärts nur im Bereiche des Uterus, vorzüglich des Collum, zu suchen hat. In sehr seltenen Fällen lassen die Spuren sich vielleicht über das Collum hinaus verfolgen, es ist aber ein Irrthum, die von Skene, Schüller und Oberdieck näher beschriebenen Gänge, welche man auch nach meinen Beobachtungen fast 
constant an der Mündung der Urethra wenigstens bei jungen Frauen findet, mit den Mündungen der Wolff'schen Gänge gleichzu stellen, wie Kocks, Böhm, Wassilieff und v. Mihalkovicz thun. Ich kann Dohrn's Ansicht bestätigen, dass die Gartner'schen Gänge im Bereiche des Uterus in der Regel in der $z$ weiten Hälfte der Schwangerschaft verschwinden, während sie bekanntlich im Bereiche der Ligamenta lata lebenslänglich wie das Par- und Epoophoron - erhalten bleiben können. Da es aber nicht ausgeschlossen ist, dass die Gartner'schen Gänge auch in dem Uterus zaweilen bestehen bleiben können, so ist es wohl möglich, dass die von Freund, G. und J. Veit, v. Preuschen u. A. beobachteten eigenthümlichen Cysten im Bereiche des Collum und des oberen Theils der Vagina derartigen Ueberbleibseln der Wolff'schen Gänge ihren Ursprung verdanken. - Es ist Zeit, dass die in gynäkologischen Aufsätzen vielfach wiederholte Irrlehre, dass die Gartner'schen Gänge sich bis zum Introitus vaginae, beziehungsweise bis zur Urethralmündung verfolgen liessen, endlich verschwinde.

\section{Die Urethra und der Damm.}

Die Ansicht Rathke's über die Bildung des Dammes im engeren Sinne des Wortes ist in mehreren Lehrbüchern und Aufsätzen durch eine von Perls stammende Erklärung über die äussere Trennung der Kloake in Darm-, Frucht- und Harngang verdrängt worden. Es findet sich, und das gilt auch für die betreffenden Originalarbeiten, keine Angabe darüber, welcher Quelle die Darstellung von Perls entnommen ist. Soviel ich habe ermitteln können, muss die von Perls in seinem Lehrbuch (II. Auflage von Neelsen, 1886, S. 630) gegebene Schilderung gemeint sein, welche aber nicht auf Beobachtung beruht, sondern lediglich eine sehematische Darstellung der obwaltenden Verhältnisse beabsichtjgt. Jeder Anatom wird mir zugeben müssen, dass weder die von Perls gegebenen 3 Abbildungen, noch die dazu gehörende Erklärung den wirklichen Verhältnissen entsprechen. Eine bläschenförmige Allantois besteht beim Menschen überhaupt nicht, auf keiner Entwicklungsstufe, und es steht mit den thatsächlichen Verhältnissen im Widerspruche, wenn Perls bei einem 5 wöchentlichen Fötus Urethra und Müller'schen Gang unmittelbar in die Allantois einmünden lässt. Perls sagt nicht, wie er sich die Entstehung der Scheidewand denkt, welche Mastdarm von dem Vestibulum 
vaginae schliesslich vollkommen trennt; es geht aber aus seiner dritten Zeichnung herror, dass er sie einfach durch Tieferwachsen des Septum recto-vaginale entstehen lässt.

Man kann sich füglich wundern, dass eine Naturbeobachtung habe einer Hypothese den Platz räumen müssen und dass letztere eine Zeit lang in gewissen Kreisen die allein gültige Ansicht gewesen ist und zum Theil noch ist.

Bei ménschlichen Embryonen von 11-13 mm Länge sieht man bei Betrachtung des Schwanzendes eine längsovale Grube, welche etwa von der Basis des Steisshöckers bis zur Spitze des Geschlechtshöckers sich erstreckt und deren Ränder verdickt sind. In diese Grube (Kloake) mündet - wie besonders an Längsschnitten deutlich zu erkennen - hinten der Darm, vor diesem der Canalis urogenitalis (Sinus urogenitalis). Zwischen beiden befindet sich ein etwa $0,3 \mathrm{~mm}$ dickes Septam. Wolff'sche und Müller'sche Gänge (Geschlechtsstrang im Sinne von Thiersch) münden hoch oben in den Canalis urogenitalis ein und kommen bei Beschreibung der Grube zunächst nicht in Betracht. Es münden also auf dieser Entwickelungstufe nur zwei Canäle (der Darm und der Canalis urogenitalis) in die Grube ein, welche, wenn man die Verhältnisse beim Erwachsenen zum Vergleiche heranzieht, von dem hinteren Rande des Anus bis zum vorderen Rande der Urethralmündung (bezw. bis zum Frenulum clitoridis) reichen würde. Die Grube verhält sich vollkommen gleich bei beiden Geschlechtern. Alsbald verkleben bei beiden Geschlechtern die epithelialen Wände des vorderen Theiles der Grube; an der verklebten Stelle sieht man äusserlich einen länglichen schmalen Wulst (Tourneux), welcher in der Regel auf der Spitze des Geschlechtshöckers mit einem Epithelhörnchen endet. Der verklebte Theil der Grube wird zu demjenigen Abschnitte der Urothra, welcher innerhalb der Glans Penis, bezw. - jedoch nur bis zu einer gewissen Entwickelungsstufe - innerhalb der Glans Clitoridis verläuft. Bei männlichen Embryonen von 6-7 cm Rumpflänge kommt es am Schlitzrande dieser epithelialen Furche zur Bildung einer Gewebsbrücke, welche die Furche zu einem an beiden Enden offenen Rohre abschliesst. Beim Weibe bleibt diese Gewebsbildung aus, der offen bleibende Schlitz verschwindet mit der Rückbildung der Glans Clitoridis ganz. Am proximalen Ende der Verklebungsstelle der Grube besteht bei beiden Geschlechtern eine rautenförmige Erweiterang, welche später an der Basis der Glans ihren 
Platz hat. Während sich bei männlichen Individuen der übrige Theil der Cloakengrube noch weiter schliesst, bleibt er bei weiblichen Individuen offen. In Folge des Längenwachthums des distalen Theils des Geschlechtsstranges kommt es dann bei weiblichen Embryonen zur Einmündung des Müller'schen Ganges in den Boden der Grube als dritter Canal. Gleichzeitig ist die Grube flacher geworden und im hinteren Theil geht die Dammbildang vor sich. Beim Manne bleibt nur der hinterste Theil der Grube offen und bildet den Anus, im Uebrigen versehwindet die Grube und zwar im mittleren Theile durch eine wirkliche mediane Verwachsung ihrer Wände. Die rautenförmige Erweiterung an der Basis der Glans ist beim Manne der letzte Abschnitt des Geschlechtsspaltes, welcher sich schliesst; bleibt dieselbe offen, so entsteht Hypospadie. Beim Weibe bleibt selbstredend die rautenförmige Erweiterung zeitlebens bestehen und bildet den zwischen Frenulum clitoridis und Orificium urethrae belegenen Theil des Vestibulum, welcher passend mit dem Namen Fossa navicularis anterior belegt werden könnte.

Die Entwickelung der Harnröhre beim Manne und Weibe bietet demnach Uebereinstimmungen und Versehiedenheiten dar; zum besseren Verständnisse der obwaltenden Vorgänge habe ich nach Präparaten die nebenstehenden schematischen Zeichnungen angefertigt, von welchen die Figuren I-III mediale Längsdurchschnitte darstellen und zwar Fig. I von Embryonen von 15-22 mm Länge, ehe die Müller'schen Gänge den Canalis urogenitalis erreicht haben und ehe die äusseren Genitalien einen Geschlechtsunterschied zeigen; Fig. II von einem weiblichen Embryo (etwa $17 \mathrm{~cm}$ Rumpflänge), nachdem der Geschlechtsstrang (die vereinigten Müller'schen Gänge) vollends herabgewachsen ist; um diese Zeit bestehen also im Wesentlichen dieselben Verhältnisse wie bei Erwachsenen. Fig. III von einem männlichen Embryo (von etwa $5 \mathrm{~cm}$ Rumpflänge) nach Zusammenwachsen der Wände des Geschlechtsspaltes (durch Schraffirung angedeutet), aber vor der Schliessung der rautenförmigen Erweiterung (R.). Fig. IV-VI geben schematische Umrisse der äusseren Genitalien und zwar: Fig. IV von Embryonen von 15-22 mm Länge (also entsprechend der Fig. I); Fig. V von einem männlichen Embryo von etwa $5 \mathrm{~cm}$ Rumpflänge (entsprechend Fig. III); Fig. VI von einem weiblichen Embryo von etwa $17 \mathrm{~cm}$ Rumpflänge (entsprechend Fig. II). In sämmtlichen Figuren bedeuten: $\mathrm{Bl}=$ Harnblase, $\mathrm{a}=$ Mündung 
der Wolff'schen Gänge (bezw. in Fig. II = Mündung der vereinịten Müller'schen Gänge, da ja bei weiblichen Embryonen die Wolff'schen Gänge frühzeitig zu Grunde gehen und an der weiteren Entwickelung des Geschlechtsstranges sich nicht betheiligen;

Fig. I.

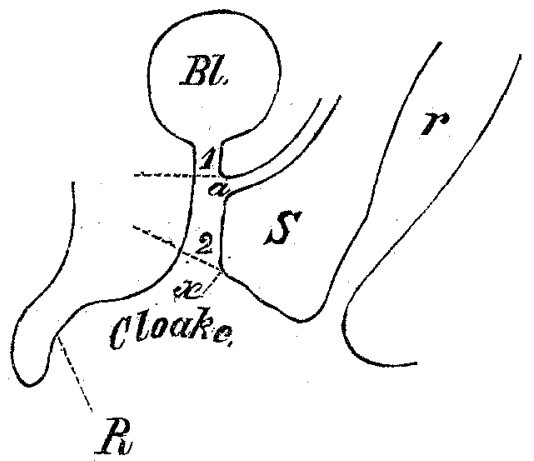

Fig. II.

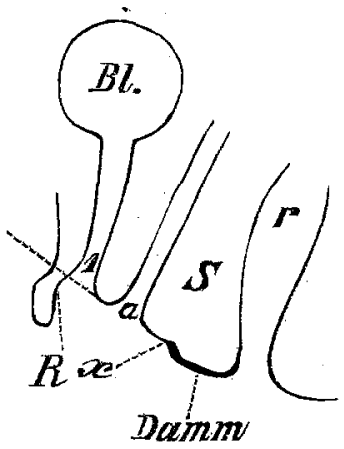

(s. S. 468), $\mathrm{S}=$ Septum recto-urogenitale bezw. in Fig. II Septum recto-genitale (vaginale); $\mathrm{R}=$ rautenförmige Erweiterung im

Hig. III.

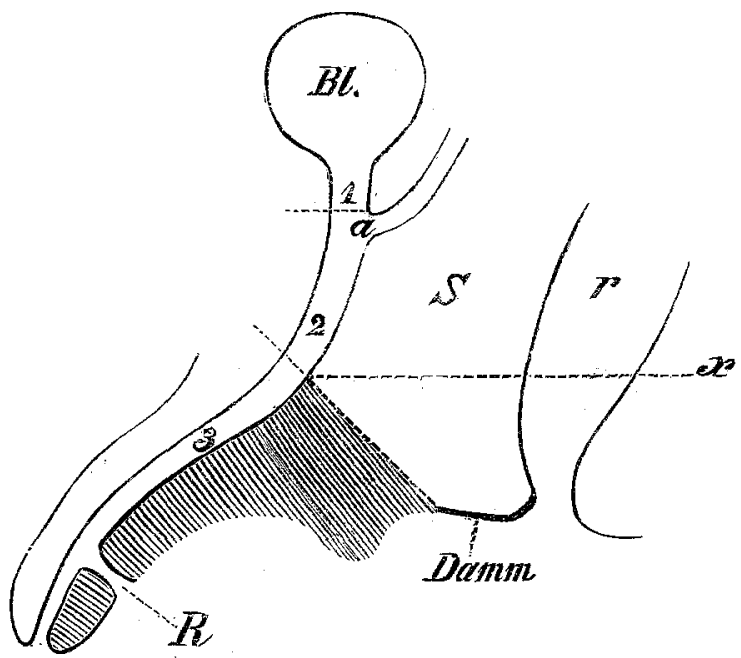

vorderen Abschnitt der Cloake; $\mathrm{v}=$ Introitus vaginae; $\mathrm{r}=$ Rectum; $\mathrm{CU}=$ Canalis urogenitalis; $\mathrm{U}=$ Urethra; $\mathrm{x}$ bezeichnet die ursprüngliche Einmündungsstelle der hinteren Wand des Canalis urogenitalis in die Cloake und deutet in den Fig. I und III an, 
wo man diese Stelle auf späteren Entwickelungsstufen zu suchen hat. Die Linien $(\alpha, \beta, \delta)$ in Fig. IV-VI sind durch gleichwerthige Punkte gezogen, deren Bedentung aus dem Text leicht ersichtlich ist; die Linie $\delta$ ist durch die, durch $x$ in Fig. I-III bezeichnete Stelle (Mündungsstelle der hinteren Wand des Canalis urogenitalis in die Cloake) gezogen. Der oberhalb der Linie $\alpha$ belegene Abschnitt der Cloake wird beim mänulichen Embryo (Fig. V) zum Eicheltheil der Urethra, beim weiblichen Embryo zur epithelialen Furche der Glans clitoridis.

Homolog sind in der Anlage bei beiden Geschlechtern die

Fig. IV.

Fig. V.

Fig. VI.

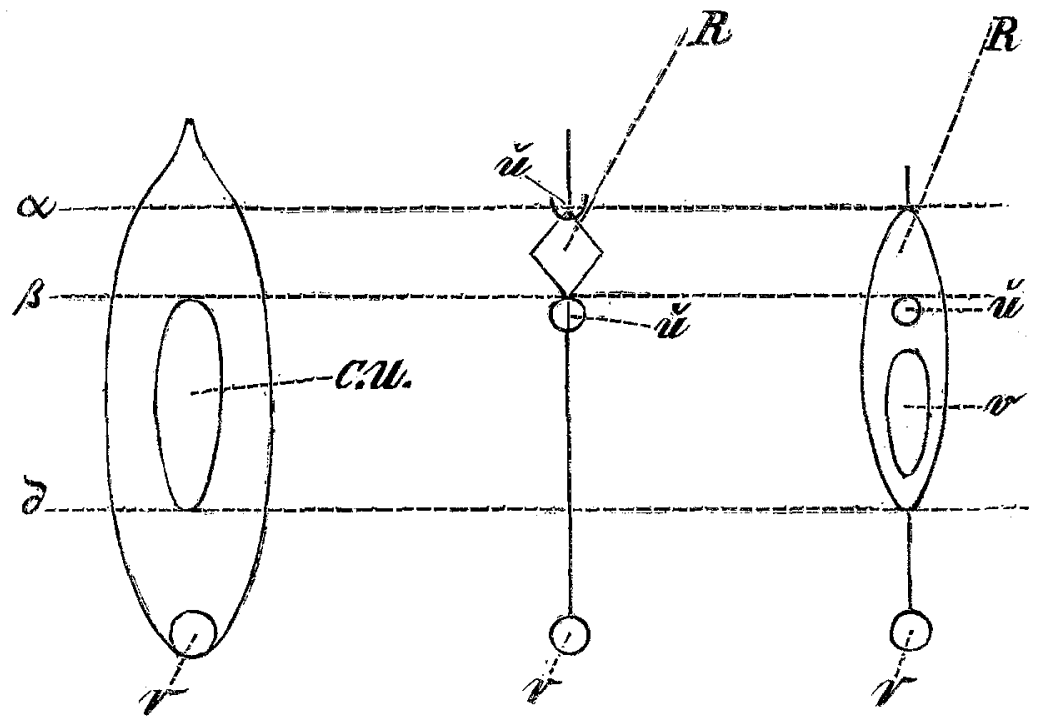

proximalen, zunächst der Blase belegenen Abschnitte der Harnröhre ( 1 in Fig. I, II und III). Die Bildung dieses proximalen Abschnittes der Harnröhre ( 1 in Fig. I) ist eng mit der Entwickelangsgeschichte der Harnblase verknüpft. Dieser Abschnitt wird nämlich durch das Hinaufrücken der Ureterenmündung gebildet, welche, wie ich an einem anderen Orte (Arch. f. mikroskop. Anatomie Bd. 34, 8. 275, 280 und 368, und Sitzungsberichte ler Königl. Preuss. Akademie der Wissenschaften, 25. Februar 1892: Ueber die Entwickelung der Harnblase beim Menschen) dargelegt habe, ursprünglich in gleicher Höhe mit den Mündungsstellen der Wolff'schen Gänge (a, Fig. I) liegen. Während aber beim Manne 
dieser Abschnitt sich vom folgenden lebenslang durch eine scharfe Grenze - Mündungsstelle der Ductus ejaculatorii (Wolff'schen Gänge, $a$ in den Fig. I und III) - trennt, verwischt sich beim Weibe diese Grenze, da die Mündung des Geschlechtsganges (vereinigte Müller'sche Gänge ohne Betheiligung der Wolff'schen Gänge) herabrückt, um später in das Vestibulum auszumünden (a in Fig. II).

Der folgende Abschnitt (2) ist anfangs bei beiden Geschlechtern gleich angelegt (Fig. I); er umfasst beim erwachsenen Manne den distalen Theil der Pars prostatica und die Pars membranacea bis zum Eintritt der Harnröhre in das Corpus cavernosum urethrae, beim Weibe verschwindet er bis auf den als Fossa navicularis posterior bekannten Abschnitt des Vestibulums. Es ist dieser Theil der ursprüngliche Canalis urogenitalis, wie ein Blick auf die Fig. I sofort zeigt; aber nur beim Manne (Fig. III) behält er den Charakter als urogenitaler Canal.

Der dritte oder cavernöse Abschnitt der Harnröhre (Fig. III, 3) kommt als solcher nor dem Manne zu und bildet sich durch Verschluss der Cloake (in Fig. III durch Schraffirung angedeatet). In seinem vorderen (Eichel-) Theile ist er allerdings, wie ich oben darlegte, auch bei weiblichen Individuen angelegt, indem die epithelialen Wände der Cloake auch bei weiblichen Embryonen in ihrem vorderen Theile mit einander verkleben. Aber nur bei männlichen Embryonen kommt es hier zu Bildung einer Röhre (in Fig. III durch Schraffirung angedeutet; siehe auch Fig. V oberhalb der Linie $\alpha$ ), bei weiblichen bleibt die Furche offen und verkleinert sich allmälig (Fig. VI oberhalb der Linie $a$ ).

Die fertige Harnröhre des Weibes (Fig. II) entspricht also nur dem Abschnitte 1 in Fig. I und III.

Zum Studium der Bildung des Dammes eignen sich nebst äusserlicher Betrachtung mittelst Loupe - am besten frontale Längsschnitte, An solchen von jüngeren Embryonen erkennt man im Bereiche des späteren Dammes die erwähnte Cloakengrube als eine $0,16 \mathrm{~mm}$ bis $0,6 \mathrm{~mm}$ tiefe Furche, deren schräg nach der Mitte zu abfallende Wände in ihrer ganzen Länge, von der Mündung des Canalis urogenitalis bis zum Anus, eine beträchtliche Verdickung ihres Epithels zeigen. Diese Verdickung betrifft jedoch nur die äussere epidermoidale Lage, deren cubische Zellen mehrfach gesehichtet sind, während die tiefe, aus Cylinderzellen bestehende Lage einreihig bleibt. An einzelnen Stellen be- 
rühren die gegenüber liegenden Epithelverdickungen einander und da der obige Befund sich bei allen Embryonen in gleicher Weise wiederholt, so ist gewiss der Schluss berechtigt, dass die erwähnten Epithelwülste den Schluss der Grube (und somit beim Weibe die Trennung des hinteren Abschnittes der Cloake in zwei Oeffnungen) herbeiführen, indem sie sammt den seitlichen Wänden der Grube mit einander verwachsen.

In welcher Weise die innere Trennung der Cloake geschieht, mit anderen Worten, durch welche Entwickelungsvorgänge der in Fig. I abgebildete Zustand geschaffen wird, darüber wissen wir bis jetzt weder beim Menschen noch bei Sängethieren etwas sicheres. Bei den jüngsten menschlichen Embryonen, die ich zu untersuchen Gelegenheit hatte ( $8 \mathrm{~mm}$ Länge) fand ich bereits den in Fig. I skizzirten Zustand - selbstredend mit Ausnahme der Gestalt der Harnblase - vor. Dagegen geht aus meinen Beobachtungen hervor, dass der Damm, im engeren Sinne des Wortes, nur durch Zusammenwachsen der beiden Seitenwände der Cloakengrube gebildet wird. Ich schliesse mich also, was den Menschen betrifft, der Ansicht Rathke's über die äussere Trennung der Cloake bei verschiedenen Wirbelthieren an, welche auch neuerdings von von Mihalkovicz und Retterer ebenfalls bei Thieren bestätigt worden ist.

Die von Reichel (Zeitschrift f. Geburtshülfe u. Gynäkologie Bd. 14) beschriebenen Analhöcker, welche ihm zufolge den Damm bilden sollen, finden sich sehr häufig, doch nicht regelmässig, bei jüngeren Embryonen, und lange nicht immer an einer bestimmten Stelle, mitunter nur auf der einen Seite; bald sitzen sie ein wenig mehr seitlich, bald ganz in der Nähe der Analöffnung, meist aber zu weit hinten, um irgend eine Bedeutung für die Bildung des Dammes haben zu können. Hierzu kommt noch, dass sie auch nach vollzogener Dammbildung sowohl ihre Lage als ihr Aussehen unverändert behalten. Möglich ist es, dass diese Gebilde ebenso wie die Fissura transversalis ani (Rathke) eine phylogenetische Bedeutung haben.

Die erste Anlage der Bartholini'schen bezw. der Cowper'sehen Drüsen habe ich in Uebereinstimmung mit Tourneux bei Embryonen von $4 \mathrm{~cm}$ Rumpflänge gefunden und zwar als eine solide schlauchähnliche Epithelausstülpung an den beiden Seitenwänden des Canalis urogenitalis, etwas oberhalb der Einmündung dieses Canals in die Cloake. Das Epithel der Drüsenanlage be- 
steht aus hohen cubischen Zellen; Verzweigungen habe ich bei Embryonen von 5-6 cm Rumpflänge zuerst gesehen. Bei grösseren Embryonen ist der inzwischen hohl gewordene Ausführungsgang der Drüsenanlage mit einem niedrigen cubischen Epithel ähnlich demjenigen des Canalis urogenitalis bekleidet, während die Verzweigungen dasselbe hohe fast cylindrische Epithel tragen, wie in der ersten Anlage. Da der ursprüngliche Canalis urogenitalis bei männlichen Embryonen erhalten bleibt und zum ansehnlichsten Abschnitte der Harnröhre sich entwickelt (s. S. 472), bei weiblichen Embryonen hingegen zum Vestibulum reducirt wird, so ist damit erklärt, wesshalb die Bartholini'schen und Cowper'schen Drüsen sammt ihren Ausmündungen, obwohl in ihrer Anlage gleichwerthig, bei erwachsenen Individuen an so verschiedenen Stellen je nach dem Geschlechte gefunden werden. (Ueber die weitere Entwickelung und feinere Anatomie dieser Drüsen s. V. Müller, Arch. f. mikroskop. Anatomie Bd. 39, 1892.)

Durch die oben geschilderten Entwickelungsvorgänge lassen sich ungezwungen alle vorkommenden Missbildungen an den äusseren Genitalien erklären, so auch dje jüngst von Reichel (a. a. 0.), Frommel (Münchener medic. Wochenschrift 1890, S. 263) und Schauta (Dieses Archiv Bd. 39, S. 484) besprochenen. In dem von Frommel beschriebenen Falle fehlte der Damm bei einem 21jährigen Mädchen vollkommen, so dass die Dammgegend das Aussehen darbot wie bei completer Dammruptur. Irgend eine Verletzung wurde in Abrede gestellt und die Mutter behauptete, dass die Genitalien ihrer Tochter von jeher so ausgesehen hätten. Hier war offenbar das Zusammenwachsen der beiden seitlichen Wülste (s. S. 475) ausgeblieben; es war also, was den Damm betrifft, ein Zustand geblieben wie in Fig. I und IV angedeutet. Bei der von Schauta behandelten Patientin hat es sich meiner Meinung nach offenbar um ein mangelhaftes Herabwachsen des distalen Abschnittes des Geschlechtsstranges (Schauta stellte auch eine Atresia vaginae fest) gehandelt (s. S. 462 u. ff.). In Folge dessen lag die Mündung der Urethra (Mündung des Canals 3 in Fig. III der Schauta'schen Abbildungen) tiefer im Vestibulum als gewöhnlich; ausserdem hatte im Bereiche des Geschlechtshöckers eine Zusammenwachsung der Ränder des Geschlechtsspaltes stattgefunden, wie sie sonst nur beim männlichen Geschlechte auftritt und zor Bildung einer kurzen Urethra distalwärts von der ursprünglichen Urethralmündung geführt. 
Die widernatürliche Verbindung zwischen Vestibulum und Rectum in den Fällen ron Reichel und Schanta lässt sich ferner dadurch erklären, dass nur in dem oberflächlichen Theile eine Verwachsung der beiden seitlichen Wülste stattgefunden hat, während die Grube in der Tiefe offen blieb, wozu noch (in Schauta's Fall) das durch die mangelhafte Entwickelung der Vagina gehemmte Herabwachsen des Septum recto-vaginale beigetragen hat. In Bezug auf Einzelheiten, Abbildungen und Literatur verweise ich ausdrücklich auf meine oben angeführten ausführlichen Veröffentlichungen. 